\title{
Mean-Variance Portfolio Optimization when each Asset has Individual Uncertain Exit-time
}

\author{
Reza Keykhaei \\ Department of Mathematics, Khansar Faculty of Mathematics \\ and Computer Science, Khansar, Iran \\ r.keykhaei@math.iut.ac.ir
}

\begin{abstract}
The standard Markowitz Mean-Variance optimization model is a single-period portfolio selection approach where the exit-time (or the time-horizon) is deterministic. In this paper the Mean-Variance portfolio selection problem has been studied with uncertain exit-time when each asset has individual uncertain exittime, which generalizes the Markowitz's model. Some conditions are provided under which the optimal portfolio of the generalized problem is independent of the exit-times distributions. Also, it is shown that under some general circumstances, the sets of optimal portfolios in the generalized model and the standard model are the same.
\end{abstract}

Keywords: Mean-Variance portfolio optimization, Optimal portfolio, Uncertain exittime, Asset uncertain exit-time.

\section{Introduction}

Portfolio management deals with the allocation of wealth among different investment opportunities in a market, considering investor's preferences on risk and return (or reward). The foundation of Modern Portfolio Theory (MPT) was established in the 1950's by (Markowitz, 1952, 1959) when he introduced his single-period Mean-Variance (M-V) optimization model. M-V portfolio selection problems seek to compute efficient portfolios. A portfolio is efficient if, with respect to its location in the M-V plane, there is no obtainable portfolio with a lower variance without a lower expected return; or a greater expected return without a greater variance. Analytical expression of the M-V efficient portfolios in the single-period case was derived by (Markowitz, 1952, 1959) and (Merton, 1972).

In fact, a single-period $\mathrm{M}-\mathrm{V}$ problem is a static optimization problem where the future price of assets are uncertain and random. But, in the real-world, there are other uncertainties, such as uncertain exit-time (or time-horizon). At first (Yaari, 1965) studied the problem of optimal consumption for an individual for which the only uncertainty in the model is the investor's time of death. (Hakansson, 1969, 1971) generalized (Yaari, 1965) to a multi-period setting with a risky asset and an uncertain time-horizon. Under the expected utility maximization framework, (Merton, 1971) studied a continuous-time optimal investment and consumption problem with uncertain exit-time which is assumed to be the first jump time of an independent Poisson process. (Liu \& Loewenstein, 2002) investigated a portfolio optimization problem with an exponentially distributed timehorizon. Recently, (Martellini \& Uroševic, 2006) have extend the standard single-period M-V model to a model with uncertain exit-time. (Huang, Zhu, Fabozzi, \& Fukushima, 2008) proposed a CVaR optimization model to deal with the case when the investment horizon is uncertain. 
(W. J. Guo \& $\mathrm{Hu}, 2005$ ) analysed a multi-period $\mathrm{M}-\mathrm{V}$ investment problem with an uncertain exit-time. (Zhang \& Li, 2012) have generalized the work of (W. J. Guo \& Hu, 2005) to the case where the asset returns are serially correlated. (Yi, Li, \& Li, 2008) studied a multi-period asset-liability management problem with uncertain investment horizon. (W. Guo \& Cai, 2013) extended models of (W. J. Guo \& Hu, 2005) and Yi et al. Error! Reference source not found. to an infinite-time horizon setting. (Wu \& Li, 2011) considered a multi-period $\mathrm{M}-\mathrm{V}$ portfolio selection problem with regime switching and uncertain exit-time. (Wu, Zeng, \& Yao, 2014) generalized (Wu \& Li, 2011) to the case when the exit-time depends on the market states. (Blanchet-Scalliet, El Karoui, Jeanblanc, \& Martellini, 2008) considered an optimal investment problem with general uncertain time-horizon.

This paper generalizes the work of (Martellini \& Uroševic, 2006). They considered two types of uncertainty in their model. The first type is asset price uncertainty and the second type is exit-time uncertainty. They assumed that all asset have the same exit-time which is the portfolio uncertain exit-time. In this paper, the single-period M-V portfolio selection problem has been studied, where each asset has individual uncertain exit-time. Some conditions are provided under which the optimal portfolio of the generalized problem is independent of the exit-times distributions. Also, it is shown that under some general circumstances the set of optimal portfolios in the generalized model coincides with the set of optimal portfolios in the standard Markowitz model.

The rest of this paper is organized as follows. The basic notations, definitions and the formulation of standard single-period $\mathrm{M}-\mathrm{V}$ portfolio selection problem are given in Section 2. In Section 3, the problem formulation is presented. Also, the main results are given. In section 4 , the random walk case is investigated. In the last section an illustrative example is provided.

\section{Standard M-V Model}

Consider a capital market with $n \geq 2$ risky assets. An investor joins the market at time $t=0$ with an initial wealth $W(0)$ and invests his/her wealth as $W(0)=\sum_{i=1}^{n} \phi_{i} S_{i}(0)$, where $\phi_{i}$ and $S_{i}(0)$ are the number of shares and the value of the $i$-th asset at the beginning of the investment period, respectively. At the end of the investment period $(t=T)$ the wealth is $W(T)=\sum_{i=1}^{n} \phi_{i} S_{i}(T)$, where $S_{i}(T)$ is the value of the $i$-th asset at the end of the period.

In the classic M-V problem the return of each asset is $r_{i}:=\frac{S_{i}(T)}{S_{i}(0)}$; and the portfolio return is

where

$$
r:=\frac{W(T)}{W(0)}=\sum_{i=1}^{n} x_{i} r_{i}
$$

$$
x_{i}=\frac{\phi_{i} S_{i}(0)}{W(0)}, \quad i=1, \ldots, n .
$$


Clearly $\sum_{i=1}^{n} x_{i}=1$. Indeed for each $i, x_{i}$ is the the assigned weight allocated to the $i$-th asset in the portfolio. Each portfolio is denoted by the column vector $\mathbf{X}=\left(x_{1}, \ldots, x_{n}\right)^{\prime} \in$ $R^{n}$. Also, let $R=\left(r_{1}, \ldots, r_{n}\right)^{\prime}$ be the random vector of returns with covariance matrix $\mathbf{V}=\left(\sigma_{i j}\right)_{n \times n}$ and mean vector $\overline{\mathbf{R}}=\left(\bar{r}_{1}, \ldots, \bar{r}_{n}\right)^{\prime}$. Here, it is assumed that $\mathbf{V}$ is positive definite, and all assets do not have the same expected return, i.e., $\overline{\mathbf{R}} \neq k \mathbf{1}$, where $\mathbf{1}$ is the $n$-column vector of ones. Obviously $\mathbb{E}(r)=\mathbf{X}^{\prime} \overline{\mathbf{R}}$ and $\operatorname{Var}(r)=\mathbf{X}^{\prime} \mathbf{V X}$. The $\mathrm{M}-\mathbf{V}$ problem for a desired return $\mu_{0}$ is:

\section{Problem 1.}

$$
\begin{array}{cl}
\min _{\mathbf{X}} & \frac{1}{2} \mathbf{X}^{\prime} \mathbf{V} \mathbf{X} \\
\text { s.t. } & \mathbf{X}^{\prime} \overline{\mathbf{R}}=\mu_{0}, \\
& \mathbf{X}^{\prime} \mathbf{1}=1 .
\end{array}
$$

If all assets have the same expected return, i.e. $\overline{\mathbf{R}}=k \mathbf{1}$, then this problem has no solution except when $\mu_{0}=k$. For this and the proof of the following theorem see chapter 4 of Error! Reference source not found..

Theorem 2.1 Problem 1 have the unique optimal solution

$$
\mathbf{X}=\frac{C-\mu_{0} B}{A C-B^{2}} \mathbf{V}^{-1} \mathbf{1}+\frac{\mu_{0} A-B}{A C-B^{2}} \mathbf{V}^{-1} \overline{\mathbf{R}},
$$

where

$$
\begin{aligned}
& A:=\mathbf{1}^{\prime} \mathbf{V}^{-1} \mathbf{1} \\
& B:=\mathbf{1}^{\prime} \mathbf{V}^{-1} \overline{\mathbf{R}}=\overline{\mathbf{R}}^{\prime} \mathbf{V}^{-1} \mathbf{1} \\
& C:=\overline{\mathbf{R}}^{\prime} \mathbf{V}^{-1} \overline{\mathbf{R}}
\end{aligned}
$$

For more investigation about properties of optimal portfolios and more details, see Error! Reference source not found..

\section{M-V Model with Assets Uncertain Exit-Times}

(Martellini \& Uroševic, 2006) introduced the concept of exit-time risk associated with an uncertain exit-time $\tau$, and show that the set of M-V optimal portfolios in the case where $\tau$ is independent of the portfolio performance and the asset returns follow a random walk, coincides with the standard case. Although, when the exit-time is dependent on asset returns, it is possible that an optimal portfolio in the standard case is not optimal in the generalized case and vice versa. They assumed that the exit-time is the same for all assets, which is the portfolio uncertain exit-time. Here the formulation of another generalized version of problem 1 is stated for which each asset has individual uncertain exit-time. Consider a person who decides to withdraw the investment in an asset before time-horizon $T$, if it has a very bad performance, to avoid more loss, and keeps the other assets. Also, consider an investor who decides to withdraw an asset from the portfolio if he faces an unexpected event. In these cases each asset has individual uncertain exit-time. Denote the portfolio exit-time by $\tau=\left(\tau_{1}, \ldots, \tau_{n}\right)^{\prime}$, where $\tau_{i}$ is the uncertain exit-time of the $i$ th asset, for $i=1, \ldots, n$. Then, the wealth at the exit-time is $W(\tau)=\sum_{i=1}^{n} \phi_{i} S_{i}\left(\tau_{i}\right)$, 
where $S_{i}\left(\tau_{i}\right)$ is the value of the $i$ th asset at its exit-time. The return of the $i$ th asset is defined as

$$
r_{i}\left(\tau_{i}\right):=\frac{S_{i}\left(\tau_{i}\right)}{S_{i}(0)}, \quad i=1, \ldots, n
$$

Let $\mathbf{R}^{\tau}=\left(r_{1}\left(\tau_{1}\right), \ldots, r_{n}\left(\tau_{n}\right)\right)^{\prime}$ be the random vector of asset returns with covariance matrix $\boldsymbol{\Sigma}$ and mean vector $\overline{\mathbf{R}}^{\tau}:=\mathbb{E}\left(\mathbf{R}^{\tau}\right)$. Now the portfolio return is

$$
r^{\tau}:=\frac{W(\tau)}{W(0)}=\sum_{i=1}^{n} \frac{\phi_{i} S_{i}(0)}{W(0)} \frac{S_{i}\left(\tau_{i}\right)}{S_{i}(0)}=\sum_{i=1}^{n} x_{i} r_{i}\left(\tau_{i}\right)
$$

Obviously $\mathbb{E}\left(r^{\tau}\right)=\mathbf{X}^{\prime} \overline{\mathbf{R}}^{\tau}$ and $\mathbb{V}$ ar $\left(r^{\tau}\right)=\mathbf{X}^{\prime} \boldsymbol{\Sigma} \mathbf{X}$. The M-V problem for the exit-time $\tau$ and a desired return $\mu$ is:

Problem 2.

$$
\begin{array}{cl}
\min _{\mathbf{X}} & \frac{1}{2} \mathbf{X}^{\prime} \boldsymbol{\Sigma} \mathbf{X} \\
\text { s.t. } & \mathbf{X}^{\prime} \overline{\mathbf{R}}^{\tau}=\mu, \\
& \mathbf{X}^{\prime} \mathbf{1}=1 .
\end{array}
$$

In the following some conditions are provided under which the generalized and the standard problems have the same set of optimal portfolios when desired mean return varies over all obtainable mean returns.

Let $g_{i}, h_{i, j}: \mathbb{R}^{m} \rightarrow \mathbb{R}$ are real-valued functions such that

$$
\begin{aligned}
& \mathbb{E}\left(r_{i}\left(\tau_{i}\right) \mid \tau_{i}=s, \tau_{j}=t\right)=\bar{r}_{i} g_{i}(s), \\
& \operatorname{Cov}\left(r_{i}\left(\tau_{i}\right), r_{j}\left(\tau_{j}\right) \mid \tau_{i}=s, \tau_{j}=t\right)=\sigma_{i j} h_{i j}(s, t),
\end{aligned}
$$

for $i, j=1, \ldots, n$. Denote $h_{i j}\left(\tau_{i}, \tau_{j}\right)$ by $h_{i}\left(\tau_{i}\right)$ for $i=j$. Then the expected return of $i$ th asset at its exit-time is

$$
\begin{aligned}
\mathbb{E}\left(r_{i}\left(\tau_{i}\right)\right) & =\int_{\mathbb{R}^{2}} \mathbb{E}\left(r_{i}\left(\tau_{i}\right) \mid \tau_{i}=s, \tau_{j}=t\right) d F(s, t) \\
& =\int_{\mathbb{R}^{2}} \bar{r}_{i} g_{i}(s) d F(s, t) \\
& =\bar{r}_{i} \mathbb{E}\left(g_{i}\left(\tau_{i}\right)\right),
\end{aligned}
$$

where $F$ denotes the distribute function corresponding to $\tau$. Using the equation

$$
\begin{aligned}
\operatorname{Cov}\left(r_{i}\left(\tau_{i}\right), r_{j}\left(\tau_{j}\right)\right) & =\mathbb{E}\left(\operatorname{Cov}\left(r_{i}\left(\tau_{i}\right), r_{j}\left(\tau_{j}\right) \mid \tau_{i}, \tau_{j}\right)\right) \\
& +\operatorname{Cov}\left(\mathbb{E}\left(r_{i}\left(\tau_{i}\right) \mid \tau_{i}, \tau_{j}\right), \mathbb{E}\left(r_{j}\left(\tau_{j}\right) \mid \tau_{i}, \tau_{j}\right)\right)
\end{aligned}
$$

the covariation between $r_{i}\left(\tau_{i}\right)$ and $r_{j}\left(\tau_{j}\right)$ can be calculated. It can be seen that 


$$
\begin{aligned}
\mathbb{E}\left(\operatorname{Cov}\left(r_{i}\left(\tau_{i}\right), r_{j}\left(\tau_{j}\right) \mid \tau_{i}, \tau_{j}\right)\right) & =\int_{\mathbb{R}^{2}} \operatorname{Cov}\left(r_{i}\left(\tau_{i}\right), r_{j}\left(\tau_{j}\right) \mid \tau_{i}=s, \tau_{j}=t\right) d F(s, t) \\
& =\int_{\mathbb{R}^{2}} \sigma_{i j} h_{i j}(s, t) d F(s, t) \\
& =\sigma_{i j} \mathbb{E}\left(h_{i j}\left(\tau_{i}, \tau_{j}\right)\right) .
\end{aligned}
$$

Also,

$$
\begin{aligned}
& \operatorname{Cov}\left(\mathbb{E}\left(r_{i}\left(\tau_{i}\right) \mid \tau_{i}, \tau_{j}\right), \mathbb{E}\left(r_{j}\left(\tau_{j}\right) \mid \tau_{i}, \tau_{j}\right)\right) \\
& =\mathbb{E}\left(\mathbb{E}\left(r_{i}\left(\tau_{i}\right) \mid \tau_{i}, \tau_{j}\right) \mathbb{E}\left(r_{j}\left(\tau_{j}\right) \mid \tau_{i}, \tau_{j}\right)\right) \\
& -\mathbb{E}\left(\mathbb{E}\left(r_{i}\left(\tau_{i}\right) \mid \tau_{i}, \tau_{j}\right)\right) \mathbb{E}\left(\mathbb{E}\left(r_{j}\left(\tau_{j}\right) \mid \tau_{i}, \tau_{j}\right)\right) \\
& =\int_{\mathbb{R}^{2}} \mathbb{E}\left(r_{i}\left(\tau_{i}\right) \mid \tau_{i}=s, \tau_{j}=t\right) \mathbb{E}\left(r_{j}\left(\tau_{j}\right) \mid \tau_{j}=t, \tau_{i}=s\right) d F(s, t) \\
& -\bar{r}_{i} \bar{r}_{j} \mathbb{E}\left(g_{i}\left(\tau_{i}\right)\right) \mathbb{E}\left(g_{j}\left(\tau_{j}\right)\right) \\
& =\int_{\mathbb{R}^{2}} \bar{r}_{i} \bar{r}_{j} g_{i}(s) g_{j}(t) d F(s, t)-\bar{r}_{i} \bar{r}_{j} \mathbb{E}\left(g_{i}\left(\tau_{i}\right)\right) \mathbb{E}\left(g_{i}\left(\tau_{i}\right)\right) \\
& =\bar{r}_{i} \bar{r}_{j} \mathbb{E}\left(g_{i}\left(\tau_{i}\right) g_{i}\left(\tau_{j}\right)\right)-\bar{r}_{i} \bar{r}_{j} \mathbb{E}\left(g_{i}\left(\tau_{i}\right)\right) \mathbb{E}\left(g_{i}\left(\tau_{i}\right)\right) \\
& =\bar{r}_{i} \bar{r}_{j} \operatorname{Cov}\left(g_{i}\left(\tau_{i}\right), g_{j}\left(\tau_{j}\right)\right) .
\end{aligned}
$$

Therefore, the covariance between the returns of asset $i$ and asset $j$ is

$$
\operatorname{Cov}\left(r_{i}\left(\tau_{i}\right), r_{j}\left(\tau_{j}\right)\right)=\sigma_{i j} \mathbb{E}\left(h_{i j}\left(\tau_{i}, \tau_{j}\right)\right)+\bar{r}_{i} \bar{r}_{j} \operatorname{Cov}\left(g_{i}\left(\tau_{i}\right), g_{j}\left(\tau_{j}\right)\right) .
$$

Assume that there exist constants $c_{1}, c_{2}$ and $c_{3}$ such that

$$
\mathbb{E}\left(g_{i}\left(\tau_{i}\right)\right)=c_{1}, \quad \mathbb{E}\left(h_{i j}\left(\tau_{i}, \tau_{j}\right)\right)=c_{2}, \quad \mathbb{C o v}\left(g_{i}\left(\tau_{i}\right), g_{j}\left(\tau_{j}\right)\right)=c_{3},
$$

for all $i, j=1, \ldots, n$. Then, it can be concluded from equations Error! Reference source not found. and (3) that

$$
\begin{aligned}
& \overline{\mathbf{R}}^{\tau}=c_{1} \overline{\mathbf{R}}, \\
& \boldsymbol{\Sigma}=c_{2} \mathbf{V}+c_{3} \mathbf{E},
\end{aligned}
$$

where $\mathbf{E}=\left(e_{i j}\right)_{n \times n}$ and $e_{i j}=\bar{r}_{i} \bar{r}_{j}$ for $i, j=1, \ldots, n$. Consequently, the mean and the variance of the portfolio return are

$$
\begin{aligned}
& \mathbb{E}\left(r^{\tau}\right)=\mathbf{X}^{\prime} \overline{\mathbf{R}}^{\tau}=c_{1} \mathbf{X}^{\prime} \overline{\mathbf{R}}, \\
& \operatorname{Var}\left(r^{\tau}\right)=\mathbf{X}^{\prime} \boldsymbol{\Sigma} \mathbf{X}=c_{2} \mathbf{X}^{\prime} \mathbf{V X}+c_{3} \mathbf{X}^{\prime} \mathbf{E X},
\end{aligned}
$$

respectively. Obviously, $\mathbb{E}(r)=\mu_{0}$ if and only if $\mu:=\mathbb{E}\left(r^{\tau}\right)=c_{1} \mu_{0}$.

Lemma 3.1 $\Sigma$ is positive definite if $c_{2}>0$.

Proof. Let $\mathbf{Y} \in \mathbb{R}^{n}$ and $\mathbf{Y} \neq \mathbf{0}$. Then

$$
\mathbf{Y}^{\prime} \mathbf{\Sigma} \mathbf{Y}=c_{2} \mathbf{Y}^{\prime} \mathbf{V} \mathbf{Y}+c_{3}\left(\overline{\mathbf{R}}^{\prime} \mathbf{Y}\right)^{2}>0,
$$

where the last inequality holds since $\mathbf{V}$ is positive definite and $c_{3}$ in non-negative (let $i=j$ in (4)). 
The following theorem has the key role in our investigation.

Theorem 3.2 If $c_{2}>0$, then problems 1 and 2 have the same unique optimal solution for which $\mu=c_{1} \mu_{0}$.

Proof. Using equation (6) the Lagrangian function corresponding to problem 2 is

$$
L\left(\mathbf{X}, \lambda_{1}, \lambda_{2}\right):=\frac{c_{2}}{2} \mathbf{X}^{\prime} \mathbf{V X}+\frac{c_{3}}{2} \mathbf{X}^{\prime} \mathbf{E X}+\lambda_{1}\left(\mathbf{X}^{\prime} \overline{\mathbf{R}}^{\tau}-\mu\right)+\lambda_{2}\left(\mathbf{X}^{\prime} \mathbf{1}-1\right) .
$$

Considering the first optimally condition we should have

$$
\begin{aligned}
& c_{2} \mathbf{V X}+c_{3} \mathbf{E X}+\lambda_{1} \overline{\mathbf{R}}^{\tau}+\lambda_{2} \mathbf{1}=\mathbf{0}, \\
& \mathbf{X}^{\prime} \overline{\mathbf{R}}^{\tau}-\mu=0, \\
& \mathbf{X}^{\prime} \mathbf{1}-1=0 .
\end{aligned}
$$

Equation (8) yields that $\mathbf{X}^{\prime} \overline{\mathbf{R}}=\mu_{0}$ and then we have $c_{1} \mathbf{E X}=\mu \overline{\mathbf{R}}$. So equation (7) can be rewritten as

$$
c_{2} \mathbf{V X}+c_{3} \frac{\mu}{c_{1}} \overline{\mathbf{R}}+\lambda_{1} c_{1} \overline{\mathbf{R}}+\lambda_{2} \mathbf{1}=\mathbf{0} .
$$

Then

$$
\mathbf{X}=\alpha \mathbf{V}^{-1} \mathbf{1}+\beta \mathbf{V}^{-1} \overline{\mathbf{R}},
$$

where

and

$$
\alpha=-\frac{\lambda_{2}}{c_{2}}
$$

$$
\beta=-\left(\frac{c_{3} \mu}{c_{2} c_{1}}+\frac{\lambda_{1} c_{1}}{c_{2}}\right) .
$$

Replacing $\mathbf{X}$ in equations (8) and (9) yields

$$
\begin{aligned}
& 1=\mathbf{1}^{\prime} \mathbf{X}=\alpha \mathbf{1}^{\prime} \mathbf{V}^{-1} \mathbf{1}+\beta \mathbf{1}^{\prime} \mathbf{V}^{-1} \overline{\mathbf{R}}=\alpha A+\beta B, \\
& \mu_{0}=\overline{\mathbf{R}}^{\prime} \mathbf{X}=\alpha \overline{\mathbf{R}}^{\prime} \mathbf{V}^{-1} \mathbf{1}+\beta \overline{\mathbf{R}}^{\prime} \mathbf{V}^{-1} \overline{\mathbf{R}}=\alpha B+\beta C .
\end{aligned}
$$

After solving the system (10)-(11) we have

$$
\alpha=\frac{C-\mu_{0} B}{A C-B^{2}}, \quad \beta=\frac{\mu_{0} A-B}{A C-B^{2}} .
$$

Now the claim follows from equations Error! Reference source not found. and Error! Reference source not found. and theorem 2.1. The uniqueness of the solution follows from positive definiteness of $\boldsymbol{\Sigma}$.

Under conditions (1), (2) and (4), theorem 3.2 guaranties that the optimal portfolio of problem 2 is independent of the exit-times distributions (see equations Error! Reference source not found. and Error! Reference source not found.). Moreover, the sets of optimal portfolios of the standard case and the generalized case are the same. 


\section{The Random Walk Case}

This section investigates the case for which the asset returns follow a random walk, i.e., for deterministic times $t$ and $s$ we have

$$
\begin{aligned}
& \mathbb{E}\left(r_{i}(t)\right)=t \bar{r}_{i}, \\
& \operatorname{Var}\left(r_{i}(t)\right)=t \sigma_{i}^{2}, \quad\left(\sigma_{i}^{2}:=\sigma_{i i}\right) \\
& \operatorname{Cov}\left(r_{i}(s), r_{j}(t)\right)=(s \wedge t) \sigma_{i j}, \quad(s \wedge t:=\min (s, t))
\end{aligned}
$$

where $\bar{r}_{i}, \sigma_{i}^{2}$ are, respectively, instantaneous mean and variance of the return of $i$ th asset, and $\sigma_{i j}$ is the instantaneous covariance between the returns of $i$ th asset and $j$ th asset. If the exit time vector $\tau$ is independent of portfolio performance then

$$
\begin{aligned}
\mathbb{E}\left(r_{i}\left(\tau_{i}\right) \mid \tau_{i}=s, \tau_{j}=t\right) & =\mathbb{E}\left(r_{i}(s) \mid \tau_{i}=s, \tau_{j}=t\right) \\
& =\mathbb{E}\left(r_{i}(s)\right) \\
& =s \bar{r}_{i} .
\end{aligned}
$$

Also,

$$
\begin{aligned}
\operatorname{Cov}\left(r_{i}\left(\tau_{i}\right), r_{j}\left(\tau_{j}\right) \mid \tau_{i}=s, \tau_{j}=t\right) & =\operatorname{Cov}\left(r_{i}(s), r_{j}(t) \mid \tau_{i}=s, \tau_{j}=t\right) \\
& =\operatorname{Cov}\left(r_{i}(s), r_{j}(t)\right) \\
& =(s \wedge t) \sigma_{i j}
\end{aligned}
$$

Therefore,

$$
\mathbb{E}\left(\operatorname{Cov}\left(r_{i}\left(\tau_{i}\right), r_{j}\left(\tau_{j}\right) \mid \tau_{i}, \tau_{j}\right)\right)=\mathbb{E}\left(\tau_{i} \wedge \tau_{j}\right) \sigma_{i j}
$$

and

$$
\begin{aligned}
\operatorname{Cov}\left(\mathbb{E}\left(r_{i}\left(\tau_{i}\right) \mid \tau_{i}, \tau_{j}\right), \mathbb{E}\left(r_{j}\left(\tau_{j}\right) \mid \tau_{i}, \tau_{j}\right)\right) & =\mathbb{E}\left(\mathbb{E}\left(r_{i}\left(\tau_{i}\right) \mid \tau_{i}, \tau_{j}\right) \mathbb{E}\left(r_{j}\left(\tau_{j}\right) \mid \tau_{i}, \tau_{j}\right)\right) \\
& -\mathbb{E}\left(\mathbb{E}\left(r_{i}\left(\tau_{i}\right) \mid \tau_{i}, \tau_{j}\right)\right) \mathbb{E}\left(\mathbb{E}\left(r_{j}\left(\tau_{j}\right) \mid \tau_{i}, \tau_{j}\right)\right) \\
& =\bar{r}_{i} \bar{r}_{j} \mathbb{E}\left(\tau_{i} \tau_{j}\right)-\bar{r}_{i} \mathbb{E}\left(\tau_{i}\right) \bar{r}_{j} \mathbb{E}\left(\tau_{j}\right) \\
& =\bar{r}_{i} \bar{r}_{j} \operatorname{Cov}\left(\tau_{i}, \tau_{j}\right) .
\end{aligned}
$$

Finally, the covariance between the returns of asset $i$ and asset $j$ is

$$
\operatorname{Cov}\left(r_{i}\left(\tau_{i}\right), r_{j}\left(\tau_{j}\right)\right)=\mathbb{E}\left(\tau_{i} \wedge \tau_{j}\right) \sigma_{i j}+\operatorname{Cov}\left(\tau_{i}, \tau_{j}\right) \bar{r}_{i} \bar{r}_{j}
$$

Theorem 4.1 Assume the asset returns follow a random walk and the exit time vector $\tau$ is independent of portfolio performance. Also, assume the expected values of $\tau_{i} \wedge \tau_{j}$ are the same for all $i$ and $j$, as well as the covariance between $\tau_{i}$ and $\tau_{j}$. Then problems 1 and 2 have the same unique optimal solution for which $\mu=c_{1} \mu_{0}$ and $c_{1}=\mathbb{E}\left(\tau_{i}\right)$ for all $i=1, \ldots, n$.

Proof. Let $g_{i}(x)=x$ and $h_{i j}(x, y)=x \wedge y$ for all $i$ and $j$. Define the constants $c_{1}, c_{2}$ and $c_{3}$ by equations (4). Obviously $c_{1}=c_{2}$ (let $i=j$ ). Now the assertion holds by Theorem 3.2 . 
Martellini and Urosevic Error! Reference source not found. assumed that all assets have the same exit-time. Theorem 4.1 is a generalization of Proposition 4 of Martellini and Urosevic Error! Reference source not found..

Corollary 4.2 (Proposition 4 of (Martellini \& Uroševic, 2006)) Assume that all assets have the same exit time $\tau$ which is independent of portfolio performance. Also, assume that the asset returns follow a random walk. Then problems 1 and 2 have the same unique optimal solution for which $\mu=\mathbb{E}(\tau) \mu_{0}$.

Proof. Note that $x \wedge x=x$. Now the proof is a obvious result of Theorem 4.1.

\section{An Illustrative Example (Dependent exit-times)}

As it is mentioned in section 3, (Martellini \& Uroševic, 2006) showed that, when the exittime is dependent on asset returns, it is possible that an optimal portfolio in the standard case is not optimal in the generalized case and vice versa, where the exit-time is the same for all assets. This section investigates a portfolio selection problem for which each asset has individual uncertain exit-time, when exit-times are dependent on assets prices. A two weeks $(T=10)$ portfolio selection problem is considered for three stocks: NEM, KO and IBM from S\&P 500. The historical stock prices are chosen from date 1/2/2013 to date $1 / 2 / 2015$. Consider an investor who is concerned about the prices. He intends to withdraw the investment in an asset in the $k$ th day if its price is strictly decreasing in the next $k$ consecutive days, and otherwise, he waits until the specified exit-time $T=10$. It is obvious that the exit-times depend on the assets prices. Figure 1 shows the efficient frontiers, that is the locus of optimal portfolios for various obtainable returns in the Mean-Variance plane, for some values of $k$. Here $k=0$ corresponds to the classic $\mathrm{M}-\mathrm{V}$ problem, and the blue curve displays the corresponding efficient frontier.

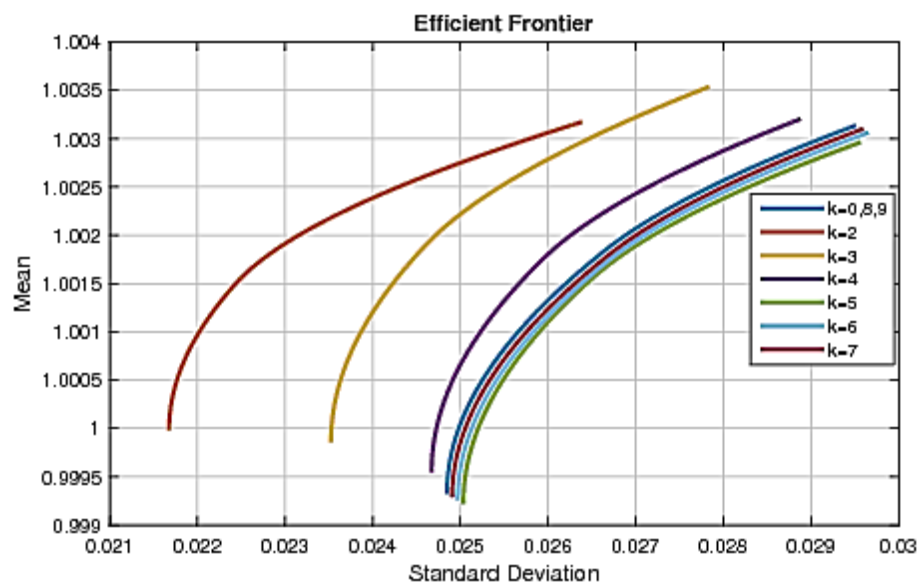

Figure 1: Efficient frontiers.

\section{References}

1. Blanchet-Scalliet, C., El Karoui, N., Jeanblanc, M., \& Martellini, L. (2008). Optimal investment decisions when time-horizon is uncertain. Journal of Mathematical Economics, 44(11), 1100-1113. 
2. Guo, W., \& Cai, J. (2013). Portfolio optimization with uncertain exit time in infinite-time horizon. Acta Mathematicae Applicatae Sinica, English Series, 29(4), 673-684.

3. Guo, W. J., \& Hu, Q. Y. (2005). Multi-period portfolio optimization when exit time is uncertain. Journal of Management Sciences in China, 8(2), 14-19.

4. Hakansson, N. H. (1969). Optimal investment and consumption strategies under risk, an uncertain lifetime, and insurance. International Economic Review, 10(3), 443-466.

5. Hakansson, N. H. (1971). Optimal entrepreneurial decisions in a completely stochastic environment. Management Science, 17(7), 427-449.

6. Huang, D., Zhu, S.-S., Fabozzi, F. J., \& Fukushima, M. (2008). Portfolio selection with uncertain exit time: a robust CVaR approach. Journal of Economic Dynamics and Control, 32(2), 594-623.

7. Liu, H., \& Loewenstein, M. (2002). Optimal portfolio selection with transaction costs and finite horizons. Review of Financial Studies, 15(3), 805-835.

8. Markowitz, H. (1952). Portfolio selection. The Journal of Finance, 7(1), 77-91.

9. Markowitz, H. (1959). Portfolio Selection, Efficent Diversification of Investments. book, J. Wiley.

10. Martellini, L., \& Uroševic, B. (2006). Static mean-variance analysis with uncertain time horizon. Management Science, 52(6), 955-964.

11. Merton, R. C. (1971). Optimum consumption and portfolio rules in a continuoustime model. Journal of Economic Theory, 3(4), 373-413.

12. Merton, R. C. (1972). An analytic derivation of the efficient portfolio frontier. Journal of Financial and Quantitative Analysis, 7(4), 1851-1872.

13. Wu, H., \& Li, Z. (2011). Multi-period mean-variance portfolio selection with Markov regime switching and uncertain time-horizon. Journal of Systems Science and Complexity, 24(1), 140-155.

14. Wu, H., Zeng, Y., \& Yao, H. (2014). Multi-period Markowitz's mean--variance portfolio selection with state-dependent exit probability. Economic Modelling, 36, 69-78.

15. Yaari, M. E. (1965). Uncertain lifetime, life insurance, and the theory of the consumer. The Review of Economic Studies, 32(2), 137-150.

16. Yi, L., Li, Z. F., \& Li, D. (2008). Multi-period portfolio selection for assetliability management with uncertain investment horizon. Journal of Industrial and Management Optimization, 4(3), 535-552.

17. Zhang, L., \& Li, Z. (2012). Multi-period mean-variance portfolio selection with uncertain time horizon when returns are serially correlated. Mathematical Problems in Engineering, 2012. 